\title{
Rigler sign: a subtle finding of pneumoperitoneum
}

\author{
Jarone Lee $\cdot$ Sapna Pawa $\cdot$ Joshua Quaas $\cdot$ \\ Kaushal H. Shah
}

Received: 11 November 2009/Accepted: 25 November 2009/Published online: 30 January 2010

(C) SIMI 2010

\section{Case report}

A 75-year-old woman with a past medical history of hypertension, presented to the emergency department (ED) with $12 \mathrm{~h}$ of persistent, non-radiating, diffuse abdominal pain. The patient had undergone a colonoscopy the day before. A chest X-ray study was done in the ED after the patient was sitting upright for at least $10 \mathrm{~min}$, but shows no free air under the diaphragm (Fig. 1). The abdominal X-ray (Fig. 2) shows air both on the inside and outside of the bowel wall consistent with free air, also known as the Rigler sign [1]. Additionally, there is a great amount of parahepatic air, outlining the lower liver edge. The Surgery service was consulted immediately for a possible bowel perforation, and the patient was taken to the operating room. Exploratory laparotomy revealed a perforated sigmoid colon. The patient underwent sigmoid colon resection, bowel re-anastomosis, and had an uncomplicated hospital course. She was discharged home in good condition.

\section{Discussion}

Perforation of the bowel is a well-documented complication of colonoscopy. Recent estimates place perforation rates after a colonoscopy at 1 in 1,400 among all patients who have a colonoscopy [2]. Fortunately, the mortality rate

J. Lee · J. Quaas · K. H. Shah ( $\square)$

St. Luke's-Roosevelt Hospital Center,

Columbia College of Physicians and Surgeons,

1111 Amsterdam Ave, New York, NY 10025, USA

e-mail: kaush.shah@gmail.com

\section{S. Pawa}

New York, NY, USA

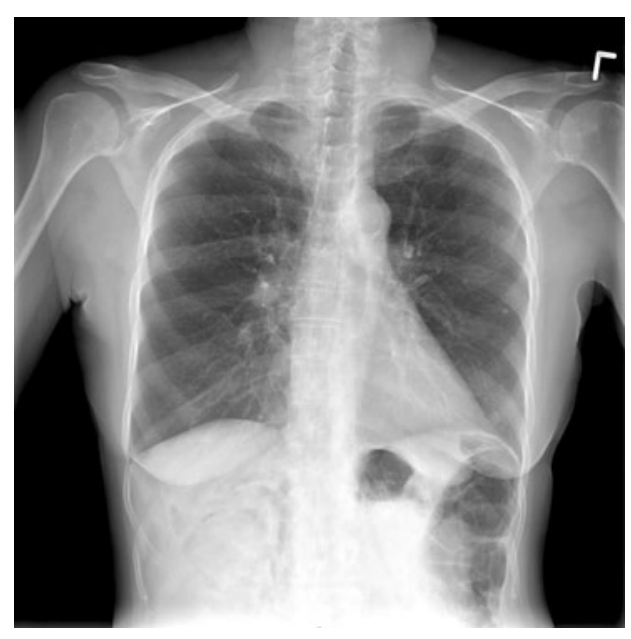

Fig. 1 Upright chest X-ray with no free air under the diaphragm

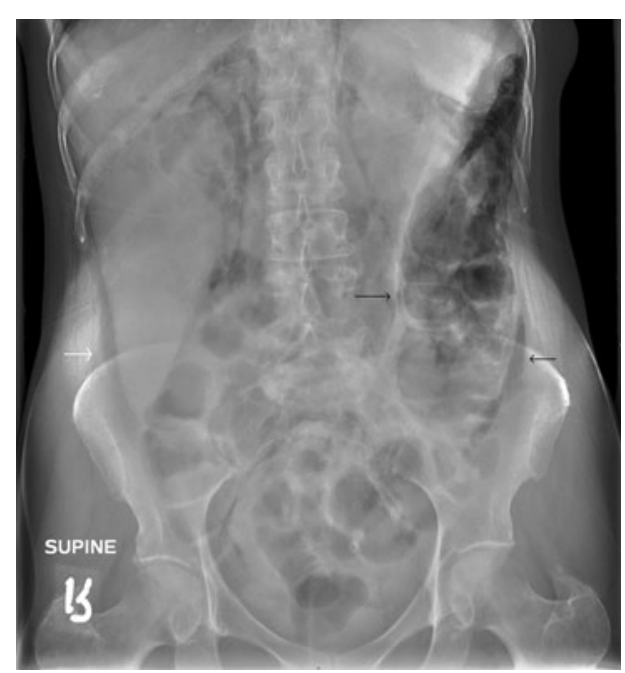

Fig. 2 Abdominal plain X-ray demonstrating two instances of Rigler sign (black arrows) and para-hepatic air (white arrow) 
is low ranging from 0 to a high of $0.02 \%$ [2]. A recent review of post-colonoscopy perforations identifies the following risk factors for perforation: (1) advanced age, (2) female gender, (3) multiple co-morbidities, (4) diverticulosis, and (5) bowel obstruction [2].

For any patient seen in the ED with gastrointestinal complaints after a recent colonoscopy, bowel perforation must be searched for. This case demonstrates that there are other signs of free air that are more subtle than free air under the diaphragm, such as the Rigler sign, but should be looked for since they can provide a diagnosis when air under the diaphragm is not seen.

On supine radiographs, the Rigler sign is the second most common sign of pneumoperitoneum. The most common finding is the presence of right upper quadrant subdiaphragmatic free air, which is typically well delineated adjacent to the liver due to differences in opacity. Other signs such as a falciform ligament sign, a football sign, or an inverted $\mathrm{V}$ sign are present in less than $3 \%$ of cases and almost always in conjunction with a positive Rigler's sign or subdiaphragmatic free air [3].

\section{Conclusion}

In conclusion, bowel perforation needs to be considered in any patient presenting with gastrointestinal complaints after a recent colonoscopy. As in our case, sometimes more subtle presentations of free air must be sought to make the diagnosis. Surgical consultation is mandatory, and the majority of these patients do very well if identified early.

Conflict of interest None.

\section{References}

1. Ly JQ (2003) The Rigler sign. Radiology 228:706-707

2. Panteris V, Haringsma J, Kuipers EJ (2009) Colonoscopy perforation rate, mechanisms and outcome: from diagnostic to therapeutic colonoscopy. Endoscopy 41:941-951

3. Levine MS, Scheiner JD, Rubesin SE et al (1991) Diagnosis of pneumoperitoneum on supine abdominal radiographs. AJR Am J Roentgenol 156:731-735 